\title{
Hypolipidemic Effect of Hyphaene thebaica (Doum-palm) in Induced Hypercholestrolemic Wistar Albino Rats
}

\author{
Nema A. Elhaj ${ }^{1}$, Nabiela M. ElBagir ${ }^{2, *}$ \\ ${ }^{1}$ Faculty of Pharmacy, Ahfad University College for Women, Sudan \\ ${ }^{2}$ Department of Biochemistry, Faculty of Veterinary Medicine, University of Khartoum, Sudan
}

Copyright $(2016$ by authors, all rights reserved. Authors agree that this article remains permanently open access under the terms of the Creative Commons Attribution License 4.0 International License

\begin{abstract}
This study is designed to investigate the effect of feeding the whole bulb (mesocarp) of Hyphaene thebaica fruit, as $3 \%$ of the diet, on lipid profile in Diet-induced hypercholesterolemic Wistar albino rats. Various dietary rations were prepared by addition of $H$. thepaica, to assess their impact on the serum triglycerides, total cholesterol and its fractions the LDL-C, HDL-C and VLDL-C in normal and induced hypercholestrolemic rats. The rats were divided into four groups and fed for three-weeks as follows: Group 1 fed basal rat diet, group 2 fed the basal rat diet mixed with $H$. thebaica, group 3 fed high fat diet (the basal rat diet mixed with, calculated amount of, egg yolk to formulate a diet of $1 \%$ cholesterol) and group 4 fed the high fat diet mixed with $H$. thebaica. By the end of the experiment a significant $(\mathrm{P}=0.03)$ increase in serum triglycerides and the VLDL-C was observed in groups ( 3 and 4 ) compared to the control group. Also the level of LDL-C increased significantly $(\mathrm{P}=0.04)$ in group 3 but decreased numerically in group 4 . Moreover, the lowest level of the total cholesterol was recorded in group 2 compared to the other groups. However, feeding $H$. thebaica when mixed with high fat in the diet, resulted in significantly $(\mathrm{P}=0.02)$ decreased HDL-C levels. In conclusion this study suggested that $H$. thebaica contains active ingredients that can manipulate blood lipids and can protect from an increase of the bad cholesterol in the blood, even if a high fat diet is consumed. The present experiment also showed that, the increase of serum triglycerides and VLDL-C levels due to feeding high fat diet in group 3, was augmented by adding $H$. thebaica to the diet in group 4 , so the study of the fatty acid composition of these lipid fractions is recommended.
\end{abstract}

Keywords Hyphaene thebaica, Lipid Profile Hypercholestrolemia

\section{Introduction}

Hyperlipidemia is a medical condition characterized by an increase in one or more of the plasma lipids, including triglycerides, cholesterol, cholesterol esters, phospholipids and or plasma lipoproteins including very low-density lipoprotein and low-density lipoprotein along with reduced high-density lipoprotein levels. This elevation of plasma lipids is among the leading risk factors associated with cardiovascular diseases [1]. Complications of hyperlipidemia include atherosclerosis, a process of arteries hardening due to deposition of cholesterol in the arterial wall, causes narrowing of the arteries, which the major cause of coronary artery disease (CAD), characterized by the accumulation of lipid and the formation of fibrous plaques within the wall of the arteries resulting in narrowing of the arteries that supply blood to the heart; Myocardial Infarction (MI): is a condition which occurs when blood and oxygen supplies are partially or completely blocked from flowing in one or more cardiac arteries, resulting in damage or death of heart cells, the occlusion may be due to ruptured atherosclerotic plaque eventually leads to heart attack [2].

In previous study the constituents of $H$. thebaica exhibited significant decrease in serum total cholesterol and Non-HDL cholesterol in rats; this was suggested to reduce the risk of atherosclerosis and subsequent cardiovascular disease[3]. The beneficial dietary hypotensive and hypolipidaemic effects of $H$. thepaica was also tested by El-Gendy [4] and they found that supplementation with Doum caused a significant decrease in systolic blood pressure, diastolic blood pressure, total cholesterol, triglyceride and LDL-C in treated patients compared to non treated ones, while they reported an increase of the HDL-C.

Hypolipidemic efficacy of $H$. thebaica was also studied in experimental nephrotic syndrome by Habib [5] and showed decrease in cholesterol, triglycerides lipoproteins "LDL, VLDL" except for HDL which showed an insignificant decrease when compared to control group, thus the water extract can reduce the hyperlipidemia in nephrotic syndrome and lead to decrease the risk of glmerulosclerosis , atherosclerosis and consequently the natural safe nontoxic $H$. thebaica fruit could be of great merit for use as hypolipidemic drug. Hetta [6] executed a 
comparative study on hypocholestrolemic effect of different fractions of $H$. thepaica (Doum) in experimental animals to investigate their effects on serum cholesterol, triglycerides and lipoproteins (HDL, cholesterol and Non HDL cholesterol), the result showed that three fractions of $H$. thepaica exhibited a highly significant decrease in serum cholesterol and Non HDL cholesterol. Decrease the Non HDL especially LDL, cholesterol can reduce the risk of atherosclerosis and subsequent cardiovascular diseases. This suggested that the plant could be of great merit for use as hypocholesterolemic drug. A comparative research on the hypoglycemic and hypolipidemic effects of some common plants extract, Aloe sinkalana, Guiera senegalensis, Trigonella foeum-graecum, Lupines albus, Zygophyllum coccineum, Allium cepa, Allium sativum, Hyphaene thebaica, Balanitesa egyptiaca and Acacia albida in type 2 diabetic patients at Eldabba area ( North sudan) carried by Kamal [7] showed a significant decrease in fasting blood sugar by plant extract of Guiera senegalensis, Allium cepa, Allium sativum, Aloe sinkalana, Whereas, Lupines albus and Hyphaene thebaica showed a significant reduction in the triglyceride and VLDL-C coupled with increased in HDL-C. Preliminary biochemical and hematological effect of aqueous suspension of pulp of Hyphaene thebaica, included changes in Blood glucose, urea albumin, triglyceride, cholesterol, total lipid, total protein, globulin, sodium, potassium and chloride ions in rats, was done by Kamis [8]. Their results revealed a significant decrease in the level of triglyceride, cholesterol, and total lipids in addition to total protein and albumin compared to control. The levels of potassium and chloride were significantly decrease and increase respectively compared to control.

\section{Objectives}

Previous studies on $H$. thebaica fruit were usually performed on various types of extracts of the fruit. Studies to investigate the effect of $H$. thebaica, as raw whole flesh (mesocarp), to test the existence of all ingredients of the fruit in their naturals concentrations and composition, on blood lipid profile is very little or lacking. The general objective of this study is to investigate the effect of feeding $H$. thebaica on serum lipids and hypercholesterolemia in experimental Wistar albino rats, by testing the effect of feeding its whole flesh, as dry powder, on lipid profile (Triglyceride, total cholesterol and its fractions the LDL-C, HDL-C and VLDL-C) of normal and hypercholestrolemic experimental animals.

\section{Materials and Methods}

\subsection{Research Design}

Randomized block design was performed.The rats were normally assigned into 4 groups of ten rats each and treated as follows: Group 1 received standard rats diet, and used as control group. Group 2 fed $H$. thebaica as $3 \%$ of the standard rat diet. Group 3 were Hypercholesterolemic rats fed the rat's diet mixed with cholesterol (calculated from egg yolk replaced w/w) as $1 \%$ of the whole diet. Group 4 were also Hypercholesterolemic rats fed the standard rats diet of $1 \%$ cholesterol but mixed with $H$. thebaica powder as $3 \%$ of the diet as in group 2. The Cholesterol was calculated as $17.41 \mathrm{mg} \backslash \mathrm{g}$ of egg yolk, according to Han, [9].

\section{Study Area}

The study was conducted in Khartoum North, in department of Biochemistry Faculty of Veterinary Medicine University of khartoum

\section{Study Period}

The study was carried during the period from January to March 2016.

\subsection{Experimental animals}

Forty Wistar albino rats with average body weight of $70 \pm 1.91 \mathrm{~g}$ were used in this study. They were kept in cages with -mesh bottom galvanized metal wall boxes under controlled environmental and nutritional conditions of about $25 \pm 2.71 \mathrm{C}^{\circ}$ and $55-60 \%$ relative humidity, in the department of Biochemistry Faculty of Veterinary Medicine University of Khartoum. They were fed standard rat diet and supplied with fresh tap water ad libitum, and kept for two weeks as adaptation period before the start of the experiment.

\section{The constituents of the rat diet}

The constituents of the basal rat diet were revised by the National Centre for Research- Khartoum Sudan. Each kg of diet consisted of: $710 \mathrm{~g}$ carbohydrates, as wheat and sohrgum (Fetarita). $167 \mathrm{~g}$ proteins, as dried minced beef meat. $120 \mathrm{~g}$ fat, as plant oil. $3 \mathrm{~g}$ table salt. The High fat diet was prepared by adding egg yolk replaced (W/W) from the rat diet calculated to supplying cholesterol as $1 \%$ of the diet.

\section{Ethical Consideration}

Ethical considerations were insured for the experimental animals. Animals were maintained under the guidelines of the research board of the Faculty of Veterinary Medicine University of Khartoum.

\subsection{Samples Collection}

Blood samples were collected from the orbital plexus of the rats for three times. The first sample was taken at time zero to record the starting values then after ten days and at day twenty-one from the start of the experiment. The serum was separated by centrifugation at 4000 r.p.m for $15 \mathrm{~min}$, and then was preserved frozen at $-20^{\circ} \mathrm{C}$ till analyzed.

\subsection{Biochemical Measurements}

Lipid profile was determined, in all groups, using 
enzymatic colorimetric methods.

Each profile consisted of:

Total cholesterol estimated according to Richmond [10].

Low density lipoprotein cholesterol (LDL-C) according to Assman [11]

High density lipoprotein cholesterol (HDL-C) according to Bachorik [12].

Very low density lipoproteins-cholesterol (VLDL-C) was calculated according to the Friedewald [13].

Triglycerides according to Cole [14].

\section{Statistical Analysis}

Data was expressed as mean SEM. The data was analyzed using SPSS windows program. The one-way analysis of variance (ANOVA) and Dunnett test was used to determine the most significant effect of feeding Hyphane thebaica. A p-value less than $0.05(\mathrm{p}<0.05)$ was considered to be significant.

\section{Results}

The effect of feeding $H$. thebaica on serum cholesterol in Wistar Albino rats, is presented in table (1). The data showed no significant difference in the serum cholesterol levels according to the type of treatment. Though, numerically, the lowest level of the total serum cholesterol was recorded in group 2, fed (Doum ) mixed with the rat diet, compared to the other groups by the end of the experiment.

Table 1. Effect of Hyphaene thebica on serum cholesterol in Wistar albino ratsmg/dl: (Mean $\pm \mathrm{SD})$

\begin{tabular}{|c|c|c|c|}
\hline $\begin{array}{c}\text { Groups } \\
\text { NO=10 }\end{array}$ & Day Zero & Day 10 & Day 21 \\
\hline G1 & $83.59 \pm 17.91$ & $83.54 \pm 23.75$ & $73.54 \pm 15.46$ \\
\hline G2 & $77.27 \pm 4.17$ & $71.73 \pm 15.13$ & $67.29 \pm 13.43$ \\
\hline G3 & $85.57 \pm 10.06$ & $77.84 \pm 14.03$ & $80.24 \pm 20.09$ \\
\hline G4 & $84.78 \pm 9.28$ & $73.68 \pm 10.18$ & $69.83 \pm 17.64$ \\
\hline Total Mean & $82.80 \pm 11.50$ & $76.70 * \pm 16.53$ & $72.73 \pm 16.92$ \\
\hline P-value & 0.06 & 0.04 & 0.07 \\
\hline
\end{tabular}

$P$. value $<0.05$ is considered to be significant*.

Table (2) presents the effect of $H$. thebica on serum Triglycerides in Wistar Albino rats which showed significantly $(\mathrm{P}=0.03)$ higher levels in serum triglycerides concentration, by the end of the experiment, in groups ( 3 and 4) compared to the control group, the two groups received high fat diet, but group (4) in addition received $H$ thebaica.

Table2. Effect of $H$. thebica on serum Triglyceride on Wistar albino rats $\mathrm{mg} / \mathrm{dl}:($ Mean $\pm \mathrm{SD})$.

\begin{tabular}{|c|c|c|c|}
\hline $\begin{array}{c}\text { Groups } \\
\text { NO=10 }\end{array}$ & Day Zero & Day 10 & Day 21 \\
\hline G1 & $50.63 \pm 21.99$ & $102.52 \pm 38.50$ & $40.45 \pm 16.09$ \\
\hline G2 & $38.79 \pm 12.23$ & $76.87 \pm 13.31$ & $54.24 \pm 15.88$ \\
\hline G3 & $71.50 \pm 20.88$ & $80.71 \pm 30.23$ & $61.8 *^{*} \pm 20.11$ \\
\hline G4 & $74.17 \pm 11.62$ & $94.80 \pm 19.38$ & $71.13^{*} \pm 9.77$ \\
\hline Total Mean & $58.77 \pm 22.35$ & $88.72 \pm 28.13$ & $56.91 \pm 19.04$ \\
\hline P-value & $\mathrm{P}=0.05$ & $\mathrm{P}=0.06$ & $\mathrm{P}=0.03$ \\
\hline
\end{tabular}

P. value $<0.05$ is considered to be significant*.

Table (3) shows the effect of $H$. thebica on serum HDL-C, a significant $(\mathrm{P}=0.02)$ decrease was seen in group (2) which fed Doum in their diet compared to the control group. Whereas, a non significantly difference of the HDL-C level was seen in group (4) which also fed Doum mixed with high fat diet.

Table3. Effect of Hyphaene thebica on serum HDL-C on Wistar albino ratsmg/dl :(Mean $\pm \mathrm{SD})$

\begin{tabular}{|c|c|c|c|}
\hline $\begin{array}{c}\text { Groups } \\
\text { NO=10 }\end{array}$ & Day Zero & Day 10 & Day 21 \\
\hline G1 & $63.85 \pm 17.77$ & $60.11 \pm 7.16$ & $54.26 \pm 9.17$ \\
\hline G2 & $56.24 \pm 56.24$ & $54.13 \pm 10.29$ & $52.72 * \pm 11.02$ \\
\hline G3 & $71.83 \pm 4.56$ & $60.71 \pm 12.80$ & $72.24 \pm 14.08$ \\
\hline G4 & $60.38 \pm 13.09$ & $49.70 \pm 8.94$ & $57.35 \pm 16.81$ \\
\hline Total time & $63.07 \pm 13.86$ & $56.16 \pm 10.66$ & $59.14 \pm 14.83$ \\
\hline P-value & 0.07 & 0.06 & 0.02 \\
\hline
\end{tabular}

$\mathrm{P}$. value $<0.05$ is considered to be significant*.

The effect of $H$. thebica on serum LDL-C in Wistar albino rats is presented in table (4). Only a significant $(\mathrm{P}=0.04)$ increase in serum LDL-C was seen in group (3) which received high fat diet, at day 10 , compared to the control group. The LDL-C in group4 which fed the H. thebica mixed with the high fat diet was not affected by the treatment.

Table 4. Effect of Hyphaene thebica on serum LDL-Cin Wistar albino rats $\mathrm{mg} / \mathrm{dl}:($ Mean $\pm \mathrm{SD})$

\begin{tabular}{|c|c|c|c|}
\hline Groups NO=10 & Day Zero & Day 10 & Day 21 \\
\hline G1 & $46.16 \pm 6.14$ & $48.36 \pm 10.13$ & $49.80 \pm 4.15$ \\
\hline G2 & $45.09 \pm 9.76$ & $43.26 \pm 9.91$ & $48.97 \pm 9.96$ \\
\hline G3 & $49.02 \pm 7.71$ & $59.55^{*} \pm 12.07$ & $57.79 \pm 12.71$ \\
\hline G4 & $53.46 \pm 10.02$ & $49.30 \pm 5.09$ & $52.68 \pm 10.92$ \\
\hline Total time & $48.43 \pm 8.85$ & $50.12 \pm 11.04$ & $52.31 \pm 10.19$ \\
\hline P-value & 0.06 & 0.04 & 0.05 \\
\hline
\end{tabular}

$\mathrm{P}$. value $<0.05$ is considered to be significant*

Table (5) shows the effect of $H$. thebica on serum VLDL-C in Wistar Albino rats, where, a significant $(\mathrm{P}=0.03)$ increase was seen in groups ( 3 and 4 ), compared to the control group at day 21 , both groups received high fat diet, but for the later the diet was mixed with Doum .

Table5. Effect of Hyphaene thebica on serum VLDL-C on Wistar albino rats:(Mean $\pm \mathrm{SD}$ )

\begin{tabular}{|c|c|c|c|}
\hline $\begin{array}{c}\text { Groups } \\
\mathrm{NO}=10\end{array}$ & Day Zero & Day 10 & Day 21 \\
\hline $\mathrm{G} 1$ & $10.12 \pm 4.39$ & $20.50 \pm 7.70$ & $8.09 \pm 3.21$ \\
\hline $\mathrm{G} 2$ & $7.75 \pm 2.44$ & $15.37 \pm 2.66$ & $10.84 \pm 3.17$ \\
\hline $\mathrm{G} 3$ & $14.30 \pm 2.44$ & $16.14 \pm 6.04$ & $12.36^{*} \pm 4.02$ \\
\hline $\mathrm{G} 4$ & $14.83 \pm 2.32$ & $18.96 \pm 3.87$ & $14.22^{*} \pm 1.95$ \\
\hline Total time & $11.75 \pm 4.47$ & $17.74 \pm 5.62$ & $11.38 \pm 3.80$ \\
\hline P-value & 0.06 & 0.06 & 0.03 \\
\hline
\end{tabular}

P. value $<0.05$ is considered to be significant

Figure1 presents the effect of feeding the powder of $H$. thebacia fruit, as $3 \%$ of the diet, on lipid profile in the 
experimental rats, for the values obtained by the end of the experimental period. The highest levels of the total cholesterol, LDL-C and the HDL-C were clearly seen in group 3 which was fed the high fat diet. Whereas, the highest levels of the triacylglycerols and the VLDL-C were reported in Group 4 which fed $H$. thebaciain addition to the high fat diet.

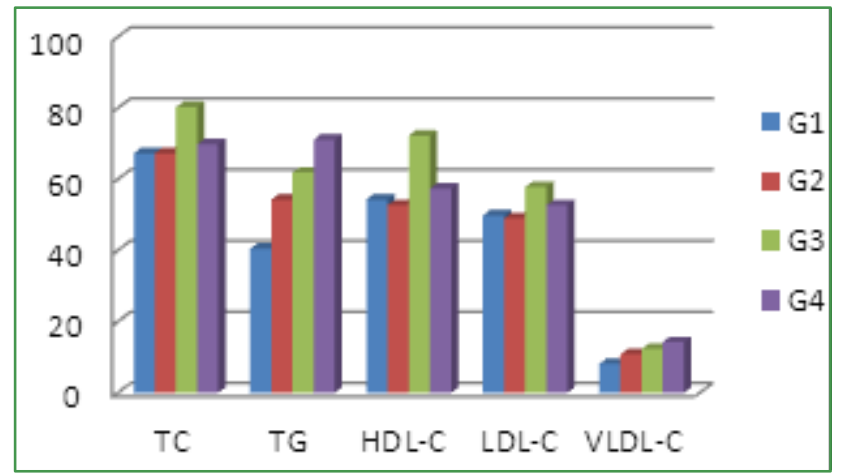

Figure 1. Effect of Hyphaene thebacia on lipid profile of hypercholesterolemic rats $\mathrm{mg} / \mathrm{dl}$.

\section{Discussion}

In human body, high levels of cholesterol, triglycerides, low density lipoprotein particles in the blood stream are strongly associated with atheroma formation in the walls of arteries (atherosclerosis), which is the principal cause of cardiovascular diseases and stroke. In contrast, high density lipoprotein (HDL) particles have been identified as a mechanism by which cholesterol can be removed from atheroma. HDL particles transport cholesterol back to the liver for excretion. Increased concentrations of HDL correlate with lower rates of atheroma progressions and even regression [5].

Effects of the characteristics of the Doum-palm fruit are not yet well studied and are still mainly anecdotal of folklore. H. thebaica is an African palm tree, common in Upper Egypt, originally native to the Nile valley, about $6 \times 5 \mathrm{~cm}$ with a shinny orange brown to deep chestnut skin (epicarp). The rind (mesocarp) in some palm is the inedible but of other it is very palatable, highly aromatic and sweet with a taste like ginger bread. H. thebaica was reported to contain important substance including saponins, tannins, and flavonoids. The fruit has natural and pharmacologic properties. H. thebaica extracts are being used in the treatment of bilharziasis, hematuria, bleeding especially after childbirth and also as hypolipidemic and hematinic suspension. The chloroform extract of the fruit improved spermatic count of male rats at low concentration but decreased it at high concentration. The tea of H. thebaica is popular in Egypt and believed to be good for diabetes. It has been also used by Egyptian people for treatment of hypertension [6].

Total serum cholesterol levels in the present study were not significantly affected by the type of treatment. Only by the end of the experimental time, the lowest level of the total cholesterol was recorded in group 2 which fed (Doum) mixed with the rat diet, compared to the other groups table (1). This may indicate a tendency, of this treatment, to reduce the serum cholesterol, if feeding continued for longer period or at a higher dose than in the present work. The two groups fed $H$. thebaica showed decreasing manner, of total cholesterol levels, during the three weeks of the experiment, although in group (4) the H. thebaica was mixed with $1 \%$ cholesterol. Whereas, serum cholesterol level was increased in group (3) which fed only the high fat diet. The overall result was presented as significant decrease, in the total mean of serum cholesterol of all groups in day ten $(\mathrm{P}=0.04)$ compared to day zero. This can be explained as a clear decreasing effect of using $H$. thebaica on the serum cholesterol level. This finding is in agreement with [3] in previous work.

In Table (2) Serum triglycerides levels were significantly $(\mathrm{P}=0.03)$ higher in the two groups fed high cholesterol diet (3) and (4) compared to the control group, hence group (4) in addition received $H$. thebaica, this indicated that Doum can increase the concentration of triglycerides in blood specially after feeding high fat diet this finding disagree with kamis [8] who reported a decreasing effect of $H$. thebica to triglycerides. In contrast to serum cholesterol, in all groups the levels of serum triglycerides were increased to very high levels after the first 10 days followed by sharp decrease at day 21.

Table (3) showed a significant decrease in serum HDL-C $(\mathrm{P}=0.02)$ in group 2 received the Doum compared to the control group. In the groups received high fat diet, numerically lower HDL-C level was observed in group (4), which fed also Doum, compared to group3. This result implied that feeding Doum might decrease the HDL-C levels in the serum. The effect of $H$. thebica on serum HDL-C in Wistar albino rats, showed a decreasing manner of the HDL-C levels in all groups except in group (3) which fed high fat diet which returned to the starting level at day zero, but no significant effect due to time in HDL-C serum concentration was observed. However, the effect of $H$. thebaica on lipid profile was studied in experimental nephrotic syndrome by Habib [5] but reported no significant effect on the HDL-C level.

In table (4), the serum LDL-C kept the same levels in the treated groups throughout the whole period of the experiment. There was no effect of time on LDL-C serum concentration due to feeding Doum throughout the 21 days of the study.

There was only significant $(\mathrm{P}=0.04)$ increase in serum LDL-C in group 3 which was fed high fat diet. Whereas, the LDL-C was not affected in group 4 which was also fed high fat diet but mixed with $H$. thebica as $3 \%$ of the meal. This finding imply that, feeding Doum, may protect against increasing the LDL-C levels even a high fat diet is consumed. Similar finding was reported by [16] that treatment of high fat diet rats with Artemisia vulgaris extract for 4 weeks at a dose of $100 \mathrm{mg} / \mathrm{kg}$ per day, resulted in normalized serum 
lipid profile.

The effect of $H$. thebaica on serum VLDL-C in Wistar Albino rats is presented in table (5) which showed a significant increase in serum VLDL-C level $(\mathrm{P}=0.03)$ in groups (3) and(4)both were received high cholesterol diet ,but the later mixed with doum. This finding indicated that feeding Doum might increase the levels of VLDL-C in the serum in hyperlipidemic subjects. In contrast in a previous work carried by Kamal [7] reported a significant reduction in the VLDL-C due to the use of $H$. thebaica extract in type 2 diabetic patients. In the present work, when the whole bulb of the fruit was used, the levels of the VLDL-C in all groups were increased after 10 days from the start of the experiment but the levels returned to the starting values at day zero without significant differences except in groups (3) and (4) which fed high fat diet. The overall values data during the period of the study showed no significant effect of time on serum concentration of the VLDL-C. This finding suggested that a comprehensive analytical study should be performed on the triglycerides and the VLDL-C blood lipid fractions, after feeding $H$. thebaica, to demonstrate their fatty acid composition.

The changes in all parameters measured, for lipid profile in this work at the end of the experimental period, were presented in figure1. Clear effect due to the use of $H$. thebaica was reported, as increased triglycerides and the VLDL-C levels, which was not seen in the total cholesterol and the LDL-C fraction.

\section{Conclusions}

The results obtained in this study showed the following: No significant change in serum total cholesterol was observed due to feeding $H$. thebaica to the experimental rats. There was an increase in serum triglycerides and the VLDL-C concentrations during the $H$. thebaica treatment and this effect increased with time so the fatty acid composition of these lipid fractions should be explored. Feeding $H$. thebaica decreased serum HDL-C levels in the tested animals. Although there was a significant increase in serum LDL-C due to feeding high fat diet, but feeding the high fat diet mixed with $H$. thebaica, showed no LDL-C increase. This finding implies that feeding $H$. thebaica may protect from the increase of the bad cholesterol, even a high fat diet is consumed.

\section{Acknowledgements}

The authors are very grateful to the technical staff at the Biochemistry lab. in the Department of Biochemistry Faculty of Veterinary Medicine University of Khartoum for their collaboration to complete this wok.

\section{REFERENCES}

[1] G. F. SHATTAT. A Review Article on Hyperlipidemia:Types, Treatments and New Drug Targets. Biomedical \& Pharmacology Journal Vol. 7(2), 399-4092014.

[2] A. Hari. Kishore, S. Ramunaik. A review on hyperlipidemic. International Journal of Novel Trends In Pharmaceutical Scienc; 3(3):59-71, 2013.

[3] H.M. Burkill. The useful plants of West Tropical Africa, 2nd (Edn), Royal Botanical garden, Kew, 4:371-373, 1997.

[4] El-Gendy, El-Mileegy, Ghyaty, Malek, El-Hamid Mousa. The Beneficial Dietary Hypotensive and Hypolipidemic Effects of Hyphaene thebaica. The Internet Journal of Alternative Medicine; 7(1):1-9, 2008.

[5] D. F. Habib, H. N. Michael, J. Y. Salib, N. M. Ahmed and M. H. Agaibyi. Hypolipidemic efficacy of Hyphaene thebaica In expermental nephrotic syndrome. International journal of pharmacy; 4(3): 28-34, 2014.

[6] M. H. Hetta, N Z, Yassin. Comparative studies on hypocholesterolemic effect on different fractions of hyphaene thebaica on experimental animal. Pharmazie Journal; 61(3):230-232, 2006.

[7] Kamal Eldin. Hypoglycemic and hypolipidemic effects of some common plants extract in type 2 diabetic patients at Eldabba area (North Sudan). Journal of pharmacy and biological science; 8(6):38-43, 2013.

[8] A. B. Kamis, S.Modu, H. Zanna, T.A. Oniyangi. Preliminary Biochemical and Haematolobical Effects of Aqueous Suspension of Pulp of Hyphaene thebaica (1) Mart In Rats. International journal of Nigerian socity for experimental biology; 13 (1): 1-7, 2003.

[9] C. K. Han,N. H. Lee. Yolk Cholesterol content In Egg from the Major Domestic Strains Of Breeding Hen. Animal Resources Lab Korea Food Research Institute Songnam-si, Kyonggi-do 462-420. Korea, 1992.

[10] W. Richmond. Preparation and Properties of a Cholesterol Oxidase from Nocardia sp. and Its Application to the Enzymatic Assay of Total Cholesterol in Serum. Clinical Chemistry, 19, 1350-1356, 1973.

[11] G.Assmann,H.-U. Jabs, U. Kohnert, W. Nolte, H. Schriewer. LDL-cholesterol determination in blood serum following precipitation of LDL with polyvinylsulfate. Clinica Chimica Acta Volume 140, Issue 1, 27 June Pages 77-83, 1984.

[12] P. S. Bachorik, K. L. Lovejoy, M. D. Carroll, C. L. Johnson. Apolipoprotein B and AI distributions in the United States, 1988-1991: results of the National Health and Nutrition Examination Survey III (NHANES III). Clin Chem. 1997 Dec; 43(12):2364-78. 1997.

[13] W. T. Friedewald, R. I. Levy, D. S. Fredrickson. Estimation of the concentration of low-density lipoprotein cholesterol in plasma, without use of the preparative ultracentrifuge. Clin Chem.; 18: 499-502, 1972.

[14] T. G. Cole,S. G.Klotzsch, J. R. McNamara. Measurement of triglyceride concentration. Handbook of Lipoprotein Testing AACC Press Washington, DC, 1997.

[15] Lippincot, Williams. Biochemistry, Wolters Kluwer Health, London, 2011.

[16] W. H. El-Tantawy. Biochemical effects, hypolipidemic and anti-inflammatory activities of Artemisia vulgaris extract in hypercholesterolemic rats. J Clin Biochem Nutr. 57(1): 33-38, 2015. 